Gemma Jiménez-Guerra ${ }^{1 *}$ Isabel Casanovas MorenoTorres $^{1 *}$

Teodora Diana Moldovan², José Maria Navarro-Mari ${ }^{1}$ José Gutiérrez-Fernández ${ }^{1,2}$

\section{Arcobacter butzleri and intestinal colonization}

'Departamento de Microbiología, Hospital Universitario Virgen de las Nieves- Instituto de Investigación Biosanitaria de Granada. Granada.

2Departamento de Microbiología, Facultad de Medicina, Universidad de Granada- Instituto de Investigación Biosanitaria de Granada. Granada.

* These authors contributed equally to this work

Article history

Received: 4 August 2019; Accepted: 5 November 2019; Published: 2 January 2020

\section{Sir,}

Arcobacter genus includes moderately curved Gram-negative bacilli, whose dimensions are comprised between 0.2 and $0.4 \mu \mathrm{m}$ of width and 1 to $3.0 \mu \mathrm{m}$ of length. The characteristic movement of these microorganisms around their own axis is also known as the 'corkscrew-like motion'. They show adequate growth in blood-enriched media, but they can multiply in many others. Their identification through the phenotypic test results difficult due to their low metabolic activity and sometimes, when using this procedure, Campylobacter spp. cannot be differentiated, thus its presence remaining underestimated [1]. Nowadays their identification (as genus or species) is usually performed using mass spectometry or molecular biology techniques [2]. Arcobacter is closely related to the Campylobacter genus, both being members of the Campylobacteriaceae family. Their main differences are the increased oxygen tolerance and the ability to grow at lower temperatures $[3,4]$. Since 2017, the Arcobacter genus encompasses a total of 26 species that have been isolated around the planet, mainly from meat and dairy products, but also from vegetables or seafood $[5,6]$. Species of the Arcobacter genus are the fourth most encountered bacteria that can be isolated from stool cultures (from patients with diarrhea) in countries like Belgium and France [7], for that reason being considered as a new emerging pathogen and as a possible zoonotic pathogen [8]. Four species have been recorded as pathogenic for humans: A. butzleri, $A$. cryaerophilus, $A$. thereius and $A$. skirrowii, and the infection produced by the first one being manifested by acute persistent diarrhea, nausea, vomiting and intestinal rhythm disturbances, although cases of bacteremia not accompanied by diarrhea have been, as well, described [7, 9-11]. No bloody diarrhea

Correspondence:

José Gutiérrez-Fernández.

Servicio de Microbiologia. Hospital Universitario Virgen de las Nieves.

Avenida de las Fuerzas Armadas, 2. E-18012 Granada, España.

E-mail: josegf@ugr.es episodes, as in the cases of infections by Campylobacter spp., have been reported. Arcobacter spp. possesses a great ability to adhere and invade the intestinal epithelium and other parts of the human body [6] leading, in some occasions, even to peritonitis. The first reported case of peritonitis caused by Arcobacter took place in 2013, when a female patient that was carrying a peritoneal dialysis catheter, acquired an infection that was not associated with gastrointestinal symptoms [12]. Although the person-to-person transmission was considered, most of the cases have been linked to the consumption of spoiled food $[4,9]$, this being also the cause of sporadic epidemic outbreaks [13]. In pig and bovine livestock, Arcobacter isolates were associated with mastitis and abortion, and it was often found as part of the normal microbiota of healthy animals [14], predominantly in birds (wild and domestic), therefore meaning that they could be an important reservoir, but it has never been reported in humans.

The aim of this study was to describe their presence in a patient, as colonizing microbiota. In February 2019, an 86-year-old female patient was hospitalized at the Traumatology and Rehabilitation Center of the Hospital Universitario Virgen de las Nieves, in Granada, in order to undergo a surgery for the implantation of a partial hip prosthesis, after a subcapital femoral fracture.

Her clinical history consisted of arterial hypertension, hypercholesterolemia, osteoporosis, Sjögren syndrome and rosacea. She was in treatment with bromazepam, amlodipine, omeprazole, ramipril, acetylsalicylic acid and she had ciprofloxacin allergy.

During former hospitalizations, she spent a long period of time in an intermediary-care center and, as it is established in the internal protocol, she previously underwent the screening for the multidrug-resistant microorganisms' colonization, which included the analysis of the colonies obtained from a rectal swab. This sample was sown following the internal protocol, in the chromogenic media CHROMID ${ }^{\circledR}$ ESBL (Bi- 

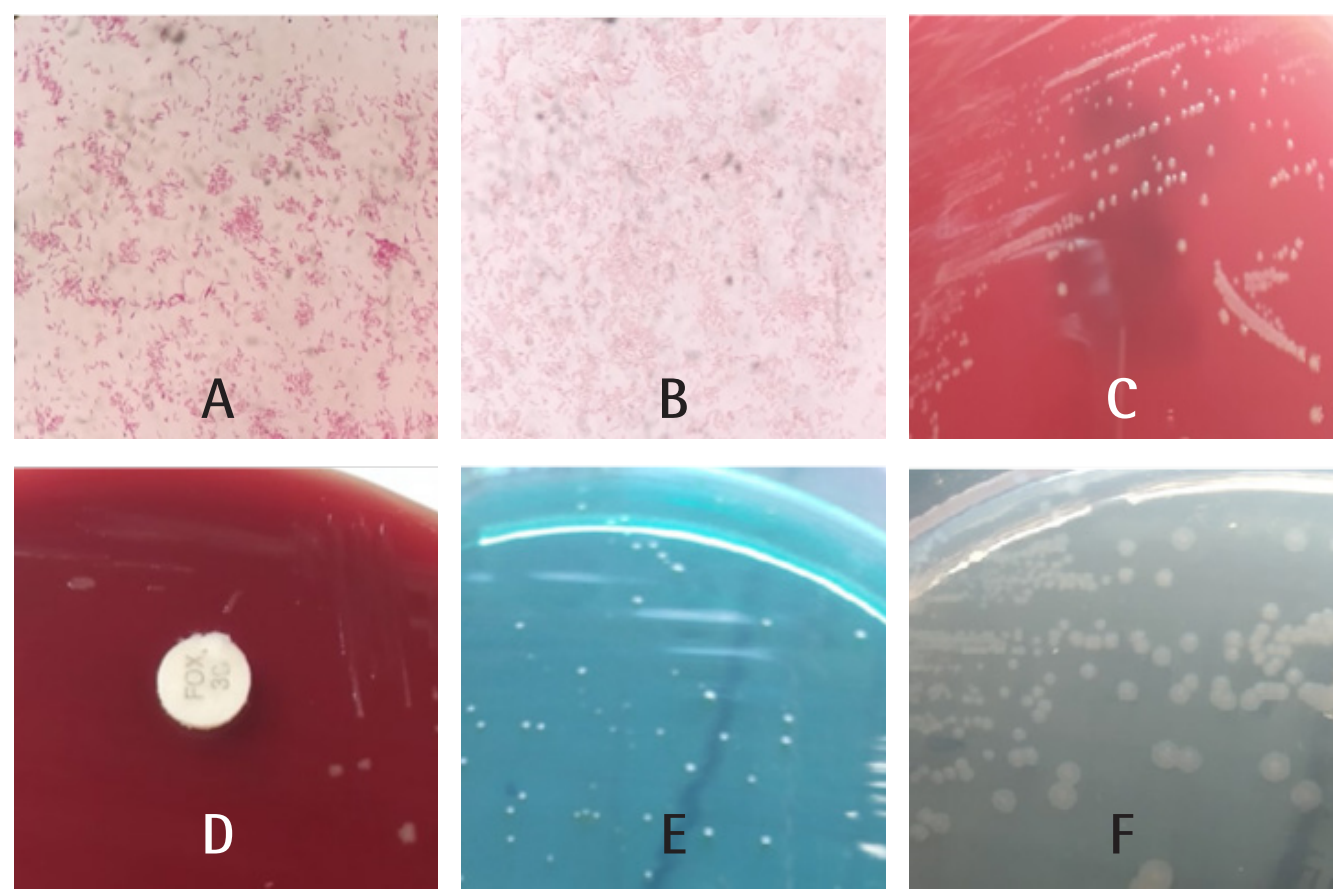

\begin{tabular}{l|l} 
Figure 1 & Microbiological study of the Arcobacter butzleri colony through Gram
\end{tabular} staining with fuchsin (A) and safranin (B), and its growing in the Columbia blood agar with $\mathrm{CO}_{2}$ (C), Campy BAP agar (D), HK agar (E) and CIN agar (F).

oMérieux, Spain) and CHROMID® MRSA SMART (BioMérieux). The first one is used for the detection of extended-spectrum beta-lactamase producing bacteria and, the second one, for the detection of methicillin-resistant Staphylococcus aureus. After $24 \mathrm{~h}$ of incubation in aerobic atmosphere at $37^{\circ} \mathrm{C}$, only in the chromogenic medium for ESBL, some green, translucent, point-shaped, round and convex colonies appeared and from these, the species identification was performed using the MALDI-TOFF mass spectrometry (Bruker Daltonics, Germany), resulting in the recognition of Arcobacter butzleri in the first four positions, with a score of 1.94 for the first, and 1.79 for the last one. A microbiological study was performed to this isolate (figure 1), which included the Gram staining, with fuchsin and safranin, and its sowing in the Columbia blood agar incubated in $\mathrm{CO}_{2}$ (Becton Dikinson, Spain), in CampyBAP agar in microaerophilic conditions, HK agar, CIN agar, XLD agar, McConkey agar and URISELECT4 (BIORAD, US), at $37^{\circ} \mathrm{C}$. No growth was identified in the last three media after 24-48h of incubation. Its antibiotic susceptibility was, as well, studied employing the E-test (MIC Strip, Liofilchem, Itlay) in Mueller-Hinton blood agar (Becton Dickinson) incubated in $\mathrm{CO}_{2}$. The obtained results are expressed in $\mathrm{mg} / \mathrm{L}$ : tetracycline 3 ; ciprofloxacin 0.064 ; trimethoprim/sulfamethoxazol $>32$; ampicillin 64; azithromycin $>256$; cefoxatime $>16 /$ cefotaxime-clavulanic acid $>1$; ceftazidime $3 /$ ceftazidime-clavulanic acid $>4$; cefotetan $>32$ / cefotetan-cloxacillin $>32$; and imipenem 2. The carbapenemase production was tested through the colorimetric assay Rapidec ${ }^{\circledR}$ Carba NP (BioMérieux), immuno- chromatography (Carba NG-Test, NG Biotech, US and OXA-23 K-Set, CorisBioConcept, Belgium) and Carba PCR (GeneXpert, Cepheid, US), and the results were negative in all the cases. The Nitrocefin beta-lactamase assay (Oxoid, UK) also turned out negative. After the isolation of this microorganism, the clinical history of the patient was reviewed, but no signs or symptoms of infectious enteritis were registered whatsoever. The patient not received antibiotherapy for treatment, the presence of $A$. butzleri was considered to be colonization, and she was discharged with no incidents during the following week. She only returned for the ordinary post-operatory check-ups.

Currently, there is not much data regarding the humans' colonization by the Arcobacter spp., and the knowledge is crucial for cases like these, when an elderly patient with such grade of immunodeficiency as the one that the Sjögren syndrome produces becomes a carrier and the microorganism does not seem to cause any harm. Even though our patient did not report any symptoms or signs during the previous months, it has been described that in patients suffering from different autoimmune diseases, the intestinal rhythm disturbances appear more frequently, being a condition that could have obscured the presence of this microorganism. Regarding a possible environmental contamination while performing the colonization study, it is important to mention that this is excluded by the consulted bibliography, since it alludes to the bacterial detection in human and animal feces as either producing gastrointestinal manifestations [6] or not [15].

The most frequently encountered pathogens producing 
infectious gastroenteritis in our media are Campylobacter and Salmonella [16], but also other potential pathogens were described [17], and they present a high percentage of strains that are susceptible to ciprofloxacin [18]. Therefore, for the majority of the cases of bacterial diarrhoea, ciprofloxacin is one of the best treatment options even though more than 10\% of the Arcobacter spp. isolates from patients with diarrhoea can be resistant [8]. In the case of our patient, even if she developed symptoms, she would never be treated with this antibiotic, due to her allergy to the fluoroquinolones, and she would probably receive tetracycline instead, due to the fact that the MIC for beta-lactams was elevated.

In elderly patients with any known underlying disease, and especially in those with autoimmune disorders, we must take into account that the behaviour of the intestinal microbiota can show substantial variations if compared to the normal population, this possibly leading to an immunomodulation that could preserve certain species for extended periods. For this reason, when a microorganism is encountered and it is already known as pathogenic but it is not causing any illness, we should remain alert.

\section{FUNDING}

None to declare.

\section{CONFLICTS OF INTEREST}

The authors declare that they have no conflicts of interest

\section{REFERENCES}

1. Prouzet-Mauleon V, Labadi L, Bouges N, Menard A, Megraud F. Arcobacter butzleri: underestimated enteropathogen. Emerg Infect Dis. 2006;12:307-9. doi: 10.3201/eid1202.050570.

2. Pentimalli D, Pegels N, Garcia T, Martin R, Gonzalez I. Specific PCR detection of Arcobacter butzleri, Arcobacter cryaerophilus, Arcobacter skirrowii, and Arcobacter cibarius in chicken meat. J Food Prot. 2009;72:1491-5. PMID: 19681276.

3. Vandamme $P$, Falsen $E_{1}$ Rossau $R$, Hoste $B$, Segers $P$, Tytgat $R$, et al. Revision of Campylobacter, Helicobacter, and Wolinella taxonomy: emendation of generic descriptions and proposal of Arcobacter gen. nov. Intern J Syst Bacteriol. 1991; 41:88-103. doi: 10.1099/00207713-41-1-88.

4. Collado L, Figueras MJ. Taxonomy, epidemiology, and clinical relevance of the genus Arcobacter. Clin Microbiol Rev. 2011; 24:17492. doi: 10.1128/CMR.00034-10.

5. Hsu $\Pi$, Lee J. Global distribution and prevalence of Arcobacter in food and water. Zoon Pub Health. 2015; 62:579-89. doi: 10.1111/ zph. 12215.

6. Ho HT, Lipman $\sqcup$, Gaastra W. Arcobacter, what is known and unknown about a potential foodborne zoonotic agent! Vet Microbiol. 2006; 115:1-13. doi: 10.1016/j.vetmic.2006.03.004.

7. Van den Abeele AM, Vogelaers D, Van Hende J, Houf K. Prevalence of Arcobacter species among humans, Belgium, 2008-2013. Emerg Infect Dis. 2014; 20:1731-4. doi: 10.3201/eid2010.140433.

8. Perez-Cataluna A, Tapiol J, Benavent C, Sarvise C, Gomez F, Martinez $B$, et al. Antimicrobial susceptibility, virulence potential and sequence types associated with Arcobacter strains recovered from human faeces. J Med Microbiol. 2017; 66:1736-43. doi: 10.1099/ jmm.0.000638.

9. Figueras MJ, Levican A, Pujol I, Ballester F, Rabada Quilez MJ, Gomez-Bertomeu F. A severe case of persistent diarrhoea associated with Arcobacter cryaerophilus but attributed to Campylobacter sp. and a review of the clinical incidence of Arcobacter spp. New Microbes New Infect. 2014; 2:31-7. doi: 10.1002/2052-2975.35.

10. Arguello E, Otto CC, Mead P, Babady NE. Bacteremia caused by Arcobacter butzleri in an immunocompromised host. J Clin Microbiol. 2015; 53:1448-51. doi: 10.1128/JCM.03450-14.

11. Houf K, Stephan R. Isolation and characterization of the emerging foodborn pathogen Arcobacter from human stool. J Microbiol Methods. 2007; 68:408-13. doi: 10.1016/j.mimet.2006.09.020.

12. Yap DY, Kwan LP, To KK, Chan TM. Arcobacter peritonitis after fluoroscopic repositioning of a Tenckhoff catheter. Perit Dial Int. 2013; 33:222-3. doi: 10.3747/pdi.2012.00114.

13. Lappi V, Archer JR, Cebelinski E, Leano F, Besser JM, Klos RF, et al. An outbreak of foodborne illness among attendees of a wedding reception in Wisconsin likely caused by Arcobacter butzleri. Foodborne Pathog Dis. 2013; 10:250-5. doi: 10.1089/fpd.2012.1307.

14. De Smet S, De Zutter L, Houf K. Spatial distribution of the emerging foodborne pathogen Arcobacter in the gastrointestinal tract of pigs. Foodborne Pathog Dis. 2012; 9:1097-103. doi: 10.1089/ fpd.2012.1184.

15. Fera MT, Russo GT, Di Benedetto A, La Camera E, Orlando A, Giandalia $A$, et al. High prevalence of Arcobacter carriage in older subjects with type 2 diabetes. J Biomed Biotechnol. 2010; 2010:489784. doi: 10.1155/2010/489784.

16. Sánchez-Capilla $A D$, Sorlózano-Puerto $A$, Rodríguez-Granger J, Martínez-Brocal A, Navarro-Marí JM, Gutiérrez-Fernández J. Infectious etiology of diarrheas studied in a third-level hospital during a five-year period. Rev Esp Enferm Dig. 2015; 107:89-97. PMID: 25659390.

17. Fernández-Fernández $E_{1}$ Martín-Rodríguez $A J$, Hernández $M$, Navarro-Mari JM, Römling U, Gutiérrez-Fernández J. First clinical isolation report of Shewanella algae from the stools of a patient with acute enteritis in Spain. Rev Esp Quimioter. 2018; 31:160-3. PMID: 29616510.

18. Guzmán-Martín JL, Navarro-Mari JM, Expósito-Ruiz M, Gutiérrez-Fernández J. Nalidixic acid surrogate test for susceptibility to ciprofloxacin in Salmonella. Revisiting the question. J Med Microbiol. 2018; 67:965-7. doi: 10.1099/jmm.0.000755. 